SU-4252-901

\title{
Non-Pauli Transitions From Spacetime Noncommutativity
}

\author{
A. P. Balachandran ${ }^{a, b} *{\text { Anosh } \operatorname{Joseph}^{a}, \dagger \text { and Pramod Padmanabhan }}^{a, \phi_{\ddagger}}$ \\ ${ }^{a}$ Department of Physics, Syracuse University, \\ Syracuse, NY 13244-1130, USA and \\ $b$ The Institute of Mathematical Sciences, \\ CIT Campus, Taramani, Chennai, India 600113
}

(Dated: May 29, 2018)

\begin{abstract}
There are good reasons to suspect that spacetime at Planck scales is noncommutative. Typically this noncommutativity is controlled by fixed "vectors" or "tensors" with numerical entries. For the Moyal spacetime, it is the antisymmetric matrix $\theta_{\mu \nu}$. In approaches enforcing Poincaré invariance, these deform or twist the method of (anti-)symmetrization of identical particle state vectors. We argue that the earth's rotation and movements in the cosmos are "sudden" events to Pauli-forbidden processes. They induce (twisted) bosonic components in state vectors of identical spinorial particles in the presence of a twist. These components induce non-Pauli transitions. From known limits on such transitions, we infer that the energy scale for noncommutativity is $\gtrsim 10^{24} \mathrm{TeV}$. This suggests a new energy scale beyond Planck scale.
\end{abstract}

\footnotetext{
*bal@phy.syr.edu

†ajoseph@phy.syr.edu

${ }^{\ddagger}$ ppadmana@syr.edu
} 


\section{INTRODUCTION}

The consideration of noncommutative spacetimes in quantum theory can be plausibly advocated from physics at the Planck scale [1]. A popular spacetime for this purpose has been the Moyal plane $\mathcal{A}_{\theta}$, a deformed spacetime algebra [2]. Here instead we consider another algebra $\mathcal{B}_{\chi \hat{n}}$ which is better suited to study atomic processes. If $\hat{x}_{\mu}$, are coordinate functions on spacetime,

$$
\hat{x}_{\mu}(x)=x_{\mu},
$$

then this algebra is defined by the relation

$$
\left[\hat{x}_{0}, \hat{x}_{j}\right]=i \chi \epsilon_{i j k} n_{i} \hat{x}_{k}
$$

where $i \in[1,2,3], \chi \in \mathbb{R}$ and $\vec{n}$ is a unit vector. Here $\chi$ and $\vec{n}$ are fixed and not dynamical. We can also introduce noncommutativity between components of $\vec{n} \wedge \vec{x}$, but we will not do So.

The Poincaré group $\mathcal{P}$ can be implemented on $\mathcal{B}_{\chi \hat{n}}$ provided the canonical coproduct $\Delta_{0}$ of the group algebra $\mathbb{C} \mathcal{P}$,

$$
\Delta_{0}(g)=g \otimes g, \quad g \in \mathcal{P}
$$

is deformed to $\Delta_{\chi \hat{n}}$ by a Drinfel'd twist $\mathcal{G}_{\chi \hat{n}}$ :

$$
\begin{gathered}
\Delta_{\chi \hat{n}}(g)=\mathcal{G}_{\chi \hat{n}}^{-1} \Delta_{0}(g) \mathcal{G}_{\chi \hat{n}} \\
\mathcal{G}_{\chi \hat{n}}=e^{-i \frac{\chi}{2}\left(P_{0} \otimes \hat{n} \cdot \vec{J}-\hat{n} \cdot \vec{J} \otimes P_{0}\right)} .
\end{gathered}
$$

For reviews see [2]. Here $P_{0}$ and $\vec{J}$ are time translation and rotation generators of $\mathcal{P}$.

Let $\mathcal{H}$ be a representation space of $\mathbb{C} \mathcal{P}$. Then we can define the flip operator $\tau_{0}$ on $\mathcal{H} \otimes \mathcal{H}$ :

$$
\tau_{0}(v \otimes w)=(w \otimes v), \quad v, w \in \mathcal{H}
$$

Since $\tau_{0}$ commutes with the action of $\Delta_{0}(g)$ on $\mathcal{H} \otimes \mathcal{H}$, we can symmetrize and antisymmetrize $\mathcal{H} \otimes \mathcal{H}$ for $\chi=0$ using the projectors $\frac{1}{2}\left(1 \pm \tau_{0}\right)$ to get untwisted bosons and fermions. But $\tau_{0}$ does not commute with the action of $\Delta_{\chi \hat{n}}$ if $\chi \neq 0$. Instead the twisted symmetrizer

$$
\begin{aligned}
& \tau_{\chi \hat{n}}=\mathcal{G}_{\chi \hat{n}}^{-1} \tau_{0} \mathcal{G}_{\chi \hat{n}}=\mathcal{G}_{\chi \hat{n}}^{-2} \tau_{0}, \\
& \mathcal{G}_{\chi \hat{n}}^{-2}=e^{i \chi\left(P_{0} \otimes \hat{n} \cdot \vec{J}-\hat{n} \cdot \vec{J} \otimes P_{0}\right)}
\end{aligned}
$$


does. The projectors $\frac{1}{2}\left(1 \pm \tau_{\chi \hat{n}}\right)$ on $\mathcal{H} \otimes \mathcal{H}$ then give the twisted bosons and fermions.

Such twisted (anti-)symmetrization can be extended to $\mathcal{H}^{\otimes k}$ for higher values of $k$.

Twisted anti-symmetrization induces $\chi \hat{n}$ dependence in energy eigenstates of electrons (nucleons) in atoms (nuclei) and corrects lifetimes in atomic (nuclear) processes. These corrections are expected to have very long time scales, $\chi$ being of the order of Planck length. (The corrections to rates from $\chi$ are $O\left(\chi^{2} E\right)$ and the corresponding times are $O\left(\chi^{-2} E^{-1}\right)$. $E$ is the typical energy involved in such transitions. Note also that we do not remark on TeV gravity till Sec.IV.) They are expected to be much longer than terrestrial times like 24 hours or 1 year. Thus the earth's motions are sudden for noncommutative effects. But the earth for example is a rotating frame and not an inertial frame. $J_{i}$ changes in that frame to $R_{i k}(t) J_{k}$ where we can permit $R(t) \in S O(3)$ to have dependence on time $t$. That changes $\hat{n} \cdot \vec{J}$ to $\hat{m} \cdot \vec{J}, m_{i}:=n_{k} R_{k i}(t)$ and hence the twisted flip operator to $\tau_{\chi \hat{m}}$. Thus effectively, the non-dynamical $\vec{n}$ gets rotated to $\vec{m}$. An effect of this sort has been noticed before by [3].

The swift change of $\tau_{\chi \hat{n}}$ to $\tau_{\chi \hat{m}}$ induces (twisted) bosonic components in multi-fermion state vectors and leads to non-Pauli effects.

We can express these effects in another manner. The energy eigenstates for the twisted flip $\tau_{\chi \hat{n}}$ depend on $\hat{n}$. When $\hat{n}$ suddenly changes to $\hat{m}$ due to the earth's motions, these states do not change in the sudden approximation. But they are not eigenstates for the flip $\tau_{\chi \hat{m}}$. When expanded in $\tau_{\chi \hat{m}}$ eigenstates, they are found to have $\tau_{\chi \hat{m}}=1$ (twisted boson) components as well. These cause the non-Pauli effects.

Such non-Pauli effects are expected for the Moyal twist $\tau_{\theta}$ as well [4].

There is a trinity of time scales, $\operatorname{atomic}\left(\tau_{-}\right)$, terrestrial $\left(\tau_{0}\right)$ and time for a Pauli-forbidden transition to $\operatorname{occur}\left(\tau_{+}\right)$, fulfilling $\tau_{-}<<\tau_{0}<<\tau_{+}$where "atomic" refers to "nuclear" as well. The earth's movements are extremely adiabatic processes for atomic dynamics and are not important (unless through Berry phase effects). But that is not the case for physics with typical times $\tau_{+}$for which the earth's motions are "sudden".

\section{THE DETAILS}

We focus on the neutral Be atom with its 4 electrons for specificity. Let $\vec{X}^{(\alpha)}(\alpha=1,2)$ be the coordinate functions of the electrons in $B e$ and $\vec{X}$ that of the nucleus. (We drop the hat on $\hat{X}^{\prime}$ s). Each of them, and hence also their differences; fulfill Eq. (2). In particular the 
relative coordinates

$$
\vec{x}^{(\alpha)}=\vec{X}^{(\alpha)}-\vec{X}
$$

fulfill

$$
\left[x_{0}, x_{j}^{(\alpha)}\right]=i \chi \epsilon_{i j k} n_{i} x_{k}^{(\alpha)} .
$$

For the Moyal plane, the relative coordinates and $x_{0}$ all mutually commute [5] forcing us to consider relativistic kinematics where center-of-mass and relative motions influence each other. That is why we consider $\mathcal{B}_{\chi \hat{n}}$.

\section{A. Preliminaries}

Let $P_{0}$ be the single electron Hamiltonian $-\frac{\vec{\nabla}^{2(\alpha)}}{2 \mu}-\frac{4 e^{2}}{\left|\vec{x}^{(\alpha)}\right|}$, where $\mu$ is the reduced mass. It represents $i \partial_{t}$ on single electron wave functions. On two-electron states, it acts as

$$
\Delta_{\chi \hat{n}}\left(P_{0}\right)=P_{0} \otimes \mathbb{I}+\mathbb{I} \otimes P_{0}
$$

$P_{0}$ commuting with $J_{i}$. As for coproducts of angular momentum $\vec{J}$, let $\hat{n}, \hat{n}^{(1)}$ and $\hat{n}^{(2)}$ form an orthonormal frame with $\hat{n}^{(1)} \wedge \hat{n}^{(2)}=\hat{n}$ and let $\vec{n}^{( \pm)}=\hat{n}^{(1)} \pm i \hat{n}^{(2)}$. Then using $\left[\hat{n} \cdot \vec{J}, \vec{n}^{( \pm)} \cdot \vec{J}\right]= \pm \vec{n}^{( \pm)} \cdot \vec{J}$, we find

$$
\begin{gathered}
\Delta_{\chi \hat{n}}(\hat{n} \cdot \vec{J})=\hat{n} \cdot \vec{J} \otimes \mathbb{I}+\mathbb{I} \otimes \hat{n} \cdot \vec{J}, \\
\Delta_{\chi \hat{n}}\left(\vec{n}^{( \pm)} \cdot \vec{J}\right)=\vec{n}^{( \pm)} \cdot \vec{J} \otimes e^{\mp i \frac{\chi}{2} P_{0}}+e^{ \pm i \frac{\chi}{2} P_{0}} \otimes \vec{n}^{( \pm)} \cdot \vec{J} .
\end{gathered}
$$

Our basic Pauli-forbidden process is that of two electrons in an excited state transiting to the ground two-electron state already occupied by two electrons. This transition can be caused by a generic perturbation $V_{\chi \vec{n}}$ of the two-electron Hamiltonian. For $\chi=0$, for simplicity, we take $V_{0}$ to be spin-independent, like the Coulomb repulsion $\frac{e^{2}}{\mid \vec{x}^{(1)}-\vec{x}^{(2)}}$, between the two electrons. As $V_{0}$ must commute with $\tau_{0}$, it is also symmetric in the electron coordinates. In the presence of the twist, the perturbation, just as $\Delta_{\chi \vec{n}}\left(P_{0}\right)$ and all observables must commute with $\tau_{\chi \vec{n}}$, making us modify $V_{0}$ to

$$
V_{\chi \vec{n}}=\frac{1}{2}\left[V_{0}+\tau_{\chi \vec{n}} V_{0} \tau_{\chi \vec{n}}\right] .
$$

So the two-electron Hamiltonian

$$
H^{(2)}=\Delta_{\chi \vec{n}}\left(P_{0}\right)+V_{\chi \vec{n}}
$$


is $\chi \vec{n}$ dependent.

Remark: Hopf symmetry, like any other symmetry can be broken. Since $H^{(2)} \neq \Delta_{\chi \vec{n}}\left(P_{0}\right)$, the Hopf symmetry generated by $P_{0}$ and $\vec{J}$ is broken.

We consider only orbital angular momentum $l=0$ energy levels for ease of calculation, and choose a basis of spin states $|\alpha\rangle_{\vec{n}}(\alpha= \pm 1$, often denoted as just \pm ) polarized in direction $\vec{n}$ :

$$
\frac{\vec{\sigma} \cdot \vec{n}}{2}|\alpha\rangle_{\vec{n}}=\frac{\alpha}{2}|\alpha\rangle_{\vec{n}}, \quad \sigma_{i}=\text { Pauli matrices. }
$$

Then if $|\nu\rangle$ are the radial single electron states for principal quantum number $\nu$, we write

$$
\begin{aligned}
|\nu\rangle \otimes|\alpha\rangle_{\vec{n}} & =|\nu, \alpha\rangle_{\vec{n}}, \\
|\nu, \alpha\rangle_{\vec{n}} \otimes\left|\nu^{\prime}, \beta\right\rangle_{\vec{n}} & =\left|\nu, \alpha ; \nu^{\prime}, \beta\right\rangle_{\vec{n}} .
\end{aligned}
$$

The energy of $|\nu, \alpha\rangle_{\vec{n}}$ is called $E_{\nu}$ :

$$
P_{0}|\nu, \alpha\rangle_{\vec{n}}=E_{\nu}|\nu, \alpha\rangle_{\vec{n}}
$$

while that of $\left|\nu, \alpha ; \nu^{\prime}, \beta\right\rangle_{\vec{n}}$, on ignoring $V_{\chi \vec{n}}$ is $E_{\nu}+E_{\nu^{\prime}}$ :

$$
\Delta_{\chi \vec{n}}\left(P_{0}\right)\left|\nu, \alpha ; \nu^{\prime}, \beta\right\rangle_{\vec{n}}=\left(E_{\nu}+E_{\nu^{\prime}}\right)\left|\nu, \alpha ; \nu^{\prime}, \beta\right\rangle_{\vec{n}}
$$

\section{B. The Ground State and Excited States}

The normalized twist-antisymmetrized two-electron ground state is

$$
\begin{aligned}
|1,1\rangle_{\chi \vec{n}} & =\sqrt{2} \frac{1-\tau_{\chi \vec{n}}}{2}\left[|1+, 1-\rangle_{\vec{n}}\right] \\
& =\frac{1}{\sqrt{2}}\left[|1+, 1-\rangle_{\vec{n}}-e^{i \chi E_{1}}|1-, 1+\rangle_{\vec{n}}\right] \\
& =-e^{i \chi E_{1}} \sqrt{2} \frac{1-\tau_{\chi \vec{n}}}{2}\left(|1-, 1+\rangle_{\vec{n}}\right) . \\
& \Delta_{\chi \vec{n}}\left(P_{0}\right)|1,1\rangle_{\chi \vec{n}}=2 E_{1}|1,1\rangle_{\chi \vec{n}}
\end{aligned}
$$

For $\chi=0$, the ground state, a spin singlet, is unique. By continuity, it remains so for $\chi \neq 0$. For this reason, replacement of either $| \pm\rangle_{\vec{n}}$ in Eq. (21) by other spin states does not give new answers.

We put two of the electrons in the above ground state. 
We put the remaining two electrons in the $s$-wave levels with $\nu=2$ and 3 . Consider for specificity their state

$$
\begin{aligned}
|2+, 3+\rangle_{\chi \vec{n}} & =\frac{1-\tau_{\chi \vec{n}}}{\sqrt{2}}|2+, 3+\rangle_{\vec{n}} \\
& =\frac{1}{\sqrt{2}}\left[|2+, 3+\rangle_{\vec{n}}-e^{i \frac{\chi}{2}\left(E_{3}-E_{2}\right)}|3+, 2+\rangle_{\vec{n}}\right]
\end{aligned}
$$

When the world turns, the Hamiltonian becomes $\Delta_{\chi \vec{m}}\left(P_{0}\right)+V_{\chi \vec{m}}$. The projectors to its

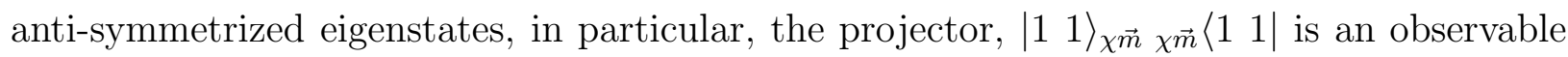
because the Hamiltonian is an observable. So in particular the Hilbert space of states contains $\mathbb{C}|1 \quad 1\rangle_{\chi \vec{m}}$.

But in the sudden approximation, it contains $|11\rangle_{\chi \vec{n}}$ as well. We now show that it is not orthogonal to the $\tau_{\chi \vec{m}}=+1$ state

$$
\frac{1+\tau_{\chi \vec{m}}}{2}|1+, 1+\rangle_{\vec{m}}=|1+, 1+\rangle_{\vec{m}}
$$

This follows from [6]

$$
\left|\vec{m}\langle\rho \mid \alpha\rangle_{\vec{n}}\right|^{2}=\frac{1}{2}\left[1+(-1)^{\frac{(\rho-\alpha)}{2}} \vec{m} \cdot \vec{n}\right]
$$

so that

$$
\left|\vec{m}\langle 1+, 1+\mid 11\rangle_{\chi \vec{n}}\right|^{2}=\frac{1}{2}\left[1-(\vec{m} \cdot \vec{n})^{2}\right] \sin ^{2}\left(\frac{\chi E_{1}}{2}\right) \neq 0
$$

if $\vec{m} \neq \vec{n}, \chi \neq 0$.

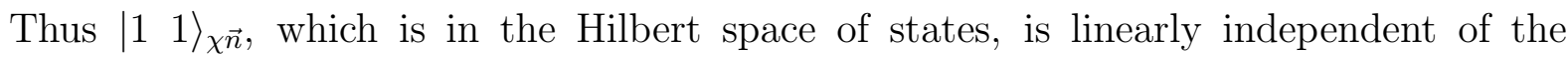
$\tau_{\chi \vec{m}}=-1$ vector $|1 \quad 1\rangle_{\chi \vec{m}}$. Hence the Hilbert space contains at least one vector with energy

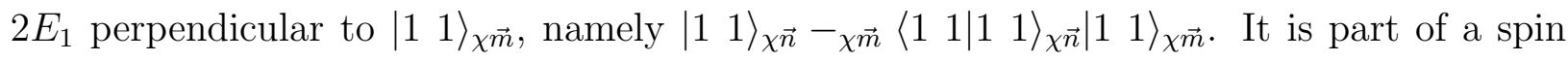
triplet. But $\Delta_{\chi \vec{m}}\left(J_{i}\right)$ are observables, form an angular momentum algebra, and its triplet representation is irreducible. So now the ground state is enhanced to contain the entire triplet of angular momentum one states. The projector

$$
\begin{gathered}
P=\mathbb{I}_{2 E_{1}}-|1,1\rangle_{\chi \vec{m} \chi \vec{m}}\langle 1,1|, \\
\mathbb{I}_{2 E_{1}}=|1,1\rangle\langle 1,1|, \\
|1,1\rangle:=|\nu=1\rangle \otimes|\nu=1\rangle
\end{gathered}
$$

to the subspace of these states is also an observable. 
We can check that $|2+, 3+\rangle_{\chi \vec{n}}$ contains bosonic components for the twist $\tau_{\chi \vec{m}}$ :

$$
\frac{1+\tau_{\chi \vec{m}}}{2}|2+, 3+\rangle_{\chi \vec{n}} \neq 0
$$

We now calculate the rate for the transition

$$
|2+, 3+\rangle_{\chi \vec{n}} \rightarrow \frac{1+\tau_{\chi \vec{m}}}{\sqrt{2}}|1 \alpha, 1 \beta\rangle_{\vec{m}}
$$

due to the potential $V_{\chi \vec{m}}$. We can neglect its $\chi$ dependence which only introduces $O\left(\chi^{2}\right)$ corrections in the transition amplitude. The perturbation $V_{0}$ is symmetric in electron coordinates as it must commute with $\tau_{0}$. By assumption, it is spin-independent.

Then if at time $t_{i}$, the two electrons were in the $|2+, 3+\rangle_{\chi \vec{n}}$ state, the transition probability $\mathcal{P}\left(t_{f}, t_{i}\right)$ to any of the three bosonic ground state at time $t_{f}$ is

$$
\begin{aligned}
\mathcal{P}\left(t_{f}, t_{i}\right) & ={ }_{\chi \vec{n}}\left\langle 2+, 3+\left|\int_{t_{i}}^{t_{f}} d \tau^{\prime} e^{-i H_{0} \tau^{\prime}} V_{0}\left(\tau^{\prime}\right) e^{i H_{0} \tau^{\prime}} P \int_{t_{i}}^{t_{f}} d \tau e^{-i H_{0} \tau} V_{0}(\tau) e^{i H_{0} \tau}\right| 2+, 3+\right\rangle_{\chi \vec{n}} \\
& =\left|\left\langle 1,1\left|\int_{t_{i}}^{t_{f}} d \tau e^{i \tau 2 E_{1}} V_{0}(\tau) e^{-i \tau\left(E_{2}+E_{3}\right)}\right| 2,3\right\rangle\right|^{2} \times P_{\mathrm{SPIN}}^{\chi}
\end{aligned}
$$

where $P$ is in Eq. (27),$\left\langle 1,1\left|V_{0}\right| 2,3\right\rangle$ is the radial matrix element of $V_{0}$ and

$$
\begin{aligned}
P_{\mathrm{SPIN}}^{\chi} & =\frac{1}{2}\left|\left(1-e^{\frac{i \chi}{2}\left(E_{3}-E_{2}\right)}\right)\right|^{2}\left[1-\frac{1}{2} \mid\left(\left.\vec{m}\left\langle+-\left|-e^{-i \chi E_{1}} \vec{m}\langle-+|\right) \mid++\right\rangle_{\vec{n}}\right|^{2}\right]\right. \\
& =\frac{1}{2}\left|\left(1-e^{i \frac{\chi}{2}\left(E_{3}-E_{2}\right)}\right)\right|^{2}\left[1-\frac{1}{2}\left|1-e^{-i \chi E_{1}}\right|^{2} \frac{1}{4}\left(1-(\vec{m} \cdot \vec{n})^{2}\right)\right]
\end{aligned}
$$

Since $\vec{m}$ and $\vec{n}$ keep changing, now average $\mathcal{P}\left(t_{f}, t_{i}\right)$ over the directions of $\vec{m}$ and $\vec{n}$ using the standard rotationally invariant measure $d \Omega=\frac{1}{4 \pi} d \cos (\theta) d \cos \phi$. Then

$$
\begin{aligned}
\left\langle m_{i}\right\rangle & \equiv \int \frac{d \Omega_{\vec{m}}}{4 \pi} m_{i}=0 \\
\left\langle m_{i} m_{j}\right\rangle & \equiv \int \frac{d \Omega_{\vec{m}}}{4 \pi} m_{i} m_{j}=\frac{1}{3} \delta_{i j}
\end{aligned}
$$

and

$$
\begin{gathered}
\left\langle\mathcal{P}\left(t_{f}, t_{i}\right)\right\rangle=\left[\left|\left\langle 1,1\left|\int_{t_{i}}^{t_{f}} d \tau e^{i \tau 2 E_{1}} V_{0}(\tau) e^{-i \tau\left(E_{2}+E_{3}\right)}\right| 2,3\right\rangle\right|^{2}\right] \times\left[\frac{1}{3}\left(5+\cos \left(\chi E_{1}\right)\right) \sin ^{2}\left(\frac{\chi}{4} \Delta E\right)\right], \\
\Delta E=E_{3}-E_{2} .
\end{gathered}
$$

Similar probabilities can be deduced for different initial and final states. The answers will not be Eq. (36), but they are still expected to be $O\left(\chi^{2}\right)$. Our bounds for $\chi$ below are not expected to change much by such changes. 
It is best to work with the branching ratio $B_{\chi}$ of the Pauli-forbidden process to an allowed process to cancel out the details specific to our model and give a formula of general applicability. The terms multiplying the $\chi$ dependent part is expected to approximate a typical Pauli-allowed process. Thus the branching ratio of a Pauli-forbidden to an allowed process is

$$
B_{\chi}=\frac{1}{3}\left[5+\cos \left(\chi E_{1}\right)\right] \sin ^{2}\left(\frac{\chi}{4} \Delta E\right) .
$$

\section{THE BOUNDS}

We can now use $B_{\chi}$ for both atomic and nuclear experiments [7-12] to deduce bounds on $\chi$, with $\Delta E$ standing for the change in energy in the nuclear or atomic transition. It also indicates whether the experiment involves nuclear or atomic transition.

The bounds are summarized in Table (II). They are obtained from the following experimental branching ratios:

Borexino [7] gives a lifetime for the process $\tau\left({ }^{12} C \rightarrow^{12} \widetilde{C}+\gamma\right)>2.1 \times 10^{27}$ years where ${ }^{12} \widetilde{C}$ is a hypothetical Pauli-forbidden nucleus with an extra nucleon in the filled $K$-shell of ${ }^{12} \mathrm{C}$. This corresponds to a branching ratio of the order of $10^{-58}$.

In the Kamiokande [8] experiment searches were made for forbidden transitions in ${ }^{16} \mathrm{O}$ nuclei and they obtain a bound on the ratio of forbidden transitions to normal transitions. This branching ratio is $<2.3 \times 10^{-57}$.

The NEMO collaboration [9] searches for anomalous ${ }^{12} \widetilde{C}$ atoms which are those with 3 $K$-shell electrons. The bound on the existence of such atoms is $\frac{12 \widetilde{C}}{12 C}<2.5 \times 10^{-12}$.

NEMO-2 [10] gives a lifetime $>4.2 \times 10^{24}$ years for a ${ }^{12} \mathrm{C}$ nuclear process which corresponds to a branching ratio of the order $<10^{-55}$.

Atomic transition experiments have been conducted in Maryland using copper $(\mathrm{Cu})$ atoms. The idea here is to introduce new electrons into a copper strip and to look for the $\mathrm{K} \mathrm{X}$-rays that would be emitted if one of these electrons were to be captured by a $\mathrm{Cu}$ atom and cascade down to the $1 S$ state despite the fact that the $1 S$ level was already filled with two electrons. The probability for this to occur was found to be less than $1.76 \times 10^{-26}[11]$.

An improved version of the experiment at Maryland has been performed by the VIP collaboration [12]. They improved the limit obtained by Ramberg and Snow at Maryland by a factor of about 40 . The limit on the probability of PEP violating interactions between 


\begin{tabular}{|c|c|c|c|}
\hline Experiment & Type & Bound on $\chi$ (Length scales) & Bound on $\chi$ (Energy scales) \\
\hline \hline Borexino & Nuclear & $\lesssim 10^{-43} \mathrm{~m}$ & $\gtrsim 10^{24} \mathrm{TeV}$ \\
Kamiokande & Nuclear & $10^{-42} \mathrm{~m}$ & $10^{23} \mathrm{TeV}$ \\
NEMO & Atomic & $10^{-12} \mathrm{~m}$ & $10^{5} \mathrm{eV}$ \\
NEMO-2 & Nuclear & $10^{-41} \mathrm{~m}$ & $10^{22} \mathrm{TeV}$ \\
Maryland & Atomic & $10^{-20} \mathrm{~m}$ & $10 \mathrm{TeV}$ \\
VIP & Atomic & $10^{-21} \mathrm{~m}$ & $100 \mathrm{TeV}$ \\
\hline
\end{tabular}

TABLE I: Bounds on the noncommutativity parameter $\chi$

external electrons and copper is found to be less than $4.5 \times 10^{-28}$.

Some of the above experiments give only lifetimes for the forbidden processes. To obtain the branching ratio in such cases we multiply the given rate with the typical lifetimes for such processes. In the case of an atomic process we use the number $10^{-16}$ seconds and for a nucler process we use $10^{-23}$ seconds.

$5.2 \times 10^{13} \mathrm{~m}^{-1}$ was the value of $\Delta E$ for ${ }^{12} C$ used in calculating the bounds on $\chi$.

The nuclear experiment by the Borexino collaboration gives the best bound for the forbidden process to date. The bound on $\chi$ obtained from this number gives a number which is much greater than Planck energy.

\section{REMARKS}

We conclude with the following remark. Corrections to the rate of a generic process from spacetime noncommutativity is $O\left(\chi^{2} E\right)$ where $E$ is a typical energy for the transition in question and $\chi^{2}$ is of the order of the square of the Planck length. The corresponding time scales are very long for conventional estimates of the Planck length and this is precisely in agreement with numbers from available experiments.

Phenomenological models of large extra dimensions (see [13] for a pedagogical introduction to models of large extra dimensions) and Randall-Sundrum scenario [14] (see also [15], intended for a general audience) bring the scale of new fundamental physics from $10^{16}$ or $10^{19} \mathrm{GeV}$ down to 10 or $100 \mathrm{TeV}$ scales. If the effective four-dimensional (reduced) Planck energy scale is in the TeV range, these time scales are very short, and may be of the order of 
$10^{-18}$ sec. So for these processes, the earth's movements are adiabatic (not sudden). By the adiabatic theorem, we expect that $\tau_{\chi \vec{n}}$ eigenstates will smoothly evolve into $\tau_{\chi \vec{m}}$ eigenstates of the same eigenvalue. No non-Pauli effects can thus be hoped for.

Lifetimes for non-Pauli transitions, which create Pauli-forbidden levels, are much longer than the age of the universe in our model. So if there were only Pauli-allowed levels at the initiation of the universe, there will not be a significant amount of non-Pauli levels now. Hence no conflict with experiment from the lack of abundance of these levels is expected.

\section{ACKNOWLEDGEMENTS}

We are very grateful to Gianpiero Mangano, who helped us with important suggestions at every stage of this work. This work has taken this shape only because of his critical comments.

A.P.B. and P.P. thank Prof. T.R. Govindarajan for the hospitality at IMSc, Chennai where this work was completed. This work was supported in part by the U.S. Department of Energy grant under the contract number DE-FG02-85ER40231. The work of A.P.B. was also supported by the Department of Science and Technology, India.

[1] S. Doplicher, K. Fredenhagen and J. E. Roberts, "Space-time quantization induced by classical gravity," Phys. Lett. B 331, 39 (1994).

[2] E. Akofor, A. P. Balachandran and A. Joseph, "Quantum Fields on the Groenewold-Moyal Plane," Int. J. Mod. Phys. A 23, 1637 (2008) arXiv:0803.4351 [hep-th]]; A. P. Balachandran, A. Joseph and P. Padmanabhan, "Causality and statistics on the Groenewold-Moyal plane," arXiv:0905.0876 [hep-th]; A. P. Balachandran and P. Padmanabhan, "The Groenewold - Moyal Plane and its Quantum Physics," arXiv:0908.3888 [hep-th].

[3] G. Piacitelli, "Twisted Covariance as a Non Invariant Restriction of the Fully Covariant DFR Model," arXiv:0902.0575 [hep-th]; L. Dabrowski, M. Godlinski and G. Piacitelli, "Lorentz Covariant k-Minkowski Spacetime," arXiv:0912.5451 [hep-th].

[4] A. P. Balachandran, G. Mangano, A. Pinzul and S. Vaidya, "Spin and statistics on the Groenwald-Moyal plane: Pauli-forbidden levels and transitions," Int. J. Mod. Phys. A 21, 
3111 (2006) arXiv:hep-th/0508002; B. Chakraborty, S. Gangopadhyay, A. G. Hazra and F. G. Scholtz, "Twisted Galilean symmetry and the Pauli principle at low energies," J. Phys. A 39, 9557 (2006) arXiv:hep-th/0601121.

[5] A. P. Balachandran and A. Pinzul, "On time-space noncommutativity for transition processes and noncommutative symmetries," Mod. Phys. Lett. A 20, 2023 (2005) arXiv:hep-th/0410199.

[6] A. P. Balachandran and P. Padmanabhan, In preparation.

[7] H. O. Back et al. [Borexino Collaboration], "New experimental limits on violations of the Pauli exclusion principle obtained with the Borexino Counting Test Facility," Eur. Phys. J. C 37, 421 (2004).

[8] Y. Suzuki et al. [Kamiokande Collaboration], "Study of invisible nucleon decay, N $\rightarrow$ neutrino neutrino anti-neutrino, and a forbidden nuclear transition in the Kamiokande detector," Phys. Lett. B 311, 357 (1993).

[9] A. S. Barabash et al., "Search for anomalous carbon atoms evidence of violation of the Pauli principle during the period of nucleosynthesis," JETP Lett. 68 (1998) 112

[10] R. Arnold et al., "Testing the Pauli exclusion principle with the NEMO-2 detector," Eur. Phys. J. A 6, 361 (1999).

[11] E. Ramberg and G. A. Snow, "A new experimental limit on small violation of the Pauli principle," Phys. Lett. B 238, 438 (1990).

[12] S. Bartalucci et al., "New experimental limit on the Pauli exclusion principle violation by electrons," Phys. Lett. B 641, 18 (2006).

[13] M. Shifman, "Large Extra Dimensions: Becoming acquainted with an alternative paradigm," Int. J. Mod. Phys. A 25, 199 (2010) arXiv:0907.3074 [hep-ph]].

[14] L. Randall and R. Sundrum, "A large mass hierarchy from a small extra dimension," Phys. Rev. Lett. 83, 3370 (1999) arXiv:hep-ph/9905221.

[15] L. Randall, "Warped Passages: Unraveling The Mysteries Of The Universe's Hidden Dimensions," New York, USA: Ecco (2005) 499 p. 